ESTUDIO DE LOS COMPORTAMIENTOS DE CIUDADANÍA ORGANIZACIONAL EN LOS PROYECTOS UNIVERSITARIOS DE RESPONSABILIDAD SOCIAL

\title{
ESTUDIO DE LOS COMPORTAMIENTOS DE CIUDADANÍA ORGANIZACIONAL EN LOS PROYECTOS UNIVERSITARIOS DE RESPONSABILIDAD SOCIAL
}

\section{BEHAVIOURAL STUDY ON ORGANIZATIONAL CITIZENSHIP IN SOCIAL RESPONSIBILITY UNIVERSITY PROJECTS}

\author{
María Mayela Terán Cázares*, Abel Partida Puente ${ }^{\star *}$, \\ Blanca Nelly Rodríguez Garza***
}

*Doctora. Universidad Autónoma de Nuevo León. Facultad de Contaduría Pública y Administración. Email: mayela.teran@gmail.com. ORCID: https://orcid.org/ 0000-0001-5089-3909

**Doctor. Universidad Autónoma de Nuevo León. Facultad de Contaduría Pública y Administración. Email: abel.partidap@uanl.mx. ORCID: https://orcid.org/ 0000-0003-3148-0548

${ }^{* * *}$ M.E. Universidad Autónoma de Nuevo León. Facultad de Contaduría Pública y Administración. Email: bngarza6@gmail.com. ORCID: https://orcid.org/ 0000-0002-9291-0292

Dirección para recibir correspondencia: mayela.teran@gmail.com 
ESTUDIO DE LOS COMPORTAMIENTOS DE CIUDADANÍA ORGANIZACIONAL EN LOS PROYECTOS UNIVERSITARIOS DE RESPONSABILIDAD SOCIAL

\section{RESUMEN}

OBJETIVO: Analizar la influencia de las dimensiones de ciudadanía organizacional en el rendimiento de los equipos participantes en proyectos de responsabilidad social.

MATERIAL Y MÉTODO: La investigación es exploratoria, descriptiva, correlacional y explicativa, con un corte transversal considerando el estudio de manera cuantitativa, cualitativa y no experimental, integrando la muestra 68 alumnos de un universo de 82 estudiantes universitarios que cursan el primer cuatrimestre en la escuela de negocios de una Universidad Privada en Monterrey, N.L.

RESULTADOS: Se encontró que para lograr el rendimiento individual y de grupo de los estudiantes universitarios en proyectos integradores de responsabilidad social universitaria, es necesario desarrollar las dimensiones de ciudadanía de ayuda, lealtad, iniciativa y autodesarrollo.

CONCLUSIONES: En las últimas décadas se ha incrementado el desarrollo de estudios relacionados con la predicción de comportamientos de la conducta humana en todo tipo de escenarios; la triple elite, organizaciones, gobierno y universidades preocupados por el futuro de la humanidad y la persistencia de comportamientos de responsabilidad social, han desarrollado estudios vinculados con aspectos humanos y operativos en donde intervienen las bases formadoras de los programas de estudio universitario, generando estrategias como el desarrollo de proyectos integradores académicos para impulsar de forma innata conductas que generen dichos comportamientos y eleven los niveles de rendimiento individual y en equipo.

PALABRAS CLAVE: Ciudadanía Organizacional. Comportamiento. Responsabilidad social. Rendimiento.

\section{ABSTRACT}

OBJECTIVE: To analyze the influence of organizational citizenship dimensions on team performance in social responsibility related projects.

MATERIAL AND METHOD: The following is a cross-sectional and correlational research with a universe of 82 university students and a sample of 68 freshmen from the Business School of a private university located in Monterrey, Nuevo Leon. 
ESTUDIO DE LOS COMPORTAMIENTOS DE CIUDADANÍA ORGANIZACIONAL EN LOS PROYECTOS UNIVERSITARIOS DE RESPONSABILIDAD SOCIAL

RESULTS: It was determined that in order to achieve either excellent individual or team performance in university students working in social responsibility related projects, it is necessary to develop the following citizenship dimensions: aid, initiative, loyalty, and selfimprovement.

CONCLUSIONS: In the last decades, the studies on human behavior related to prediction have increased in different scenarios; the triple elite, organizations, government and universities, concerned about the future of humankind as well as the endurance of social responsibility behavior, have carried out studies related to human and operational aspects entrusted to university curricula, thus generating strategies in order to develop scholar integrative projects and also to foster innate conducts which in return will increase performance levels both individually or collectively.

KEY WORDS: Organizational citizenship. Behavior. Social responsibility. Endurance.

\section{INTRODUCCIÓN}

Uno de los propósitos de la Organización de las Naciones Unidas para la Educación, la Ciencia y la Cultura (UNESCO) en la actualidad, es que las Instituciones Educativas se comprometan a formar ciudadanos que se interesen en crear un futuro más sostenible. Para ello, es conveniente imaginar primero cómo sería un mundo sostenible, siendo este el gran paradigma de las Naciones Unidas y debiera formar parte de la visión de las escuelas y facultades de negocios.

En las últimas décadas del siglo $X X$, se empezó a vislumbrar una preocupación por el futuro de la humanidad, de la vida animal y vegetal, y por los recursos imprescindibles para la vida. Lo anterior, debido a la relación entre el acelerado crecimiento económico y sus efectos ocasionados a nuestro medio ambiente, lo que hizo reflexionar sobre el peligro de la supervivencia de la especie humana (UNESCO, 2012). El crecimiento económico predominante a nivel mundial, conduce inevitablemente al agotamiento de los recursos naturales de nuestro planeta de forma paulatina, así como a la degradación ambiental y al aumento de la pobreza, lo que hace pensar en la idea de falta de solidaridad entre las generaciones. 
ESTUDIO DE LOS COMPORTAMIENTOS DE CIUDADANÍA ORGANIZACIONAL EN LOS PROYECTOS UNIVERSITARIOS DE RESPONSABILIDAD SOCIAL

De acuerdo con la UNESCO (2016), "EI desarrollo sostenible fue definido en el informe de Bruntland, en 1987, como un desarrollo que satisface las necesidades de la generación presente, sin comprometer la capacidad de las generaciones futuras de satisfacer sus propias necesidades". En dicho informe se plantea la necesidad de una participación más efectiva de los ciudadanos para unificar los propósitos del desarrollo económico y social, con la preservación del medio ambiente, a través de una distribución más racional y equitativa de los recursos.

La educación formal, impartida en Instituciones de Educación Superior (IES), debe complementar los contenidos propios de formación profesional, con lecciones de vida (experiencias) y el empirismo (la práctica) del colectivo social, lo cual traerá resultados de aplicación inmediata (Rodríguez, 2013). Al mismo tiempo, es responsabilidad de las escuelas, la generación de conocimiento en torno al medio ambiente y la formación de competencias en los estudiantes que los hagan reflexionar sobre la protección, conservación y utilización racional de los recursos naturales. En tal sentido, es imperante un modelo de educación para el desarrollo sustentable, donde los docentes generen de forma inter y multidisciplinaria acciones y proyectos, para que los estudiantes participen activamente y de manera colaborativa.

No es responsabilidad exclusiva de los gobiernos, las personas, las empresas, la sociedad civil, las instituciones educativas, deben convertirse en los agentes responsables de lograr un futuro más sostenible; y todos deben contribuir a su manera. Como señala Cajiga (2004), "la Responsabilidad Social Empresarial (RSE) es inherente a la empresa, recientemente se ha convertido en una nueva forma de gestión y de hacer negocios, en la cual la empresa se ocupa de que sus operaciones sean sustentables en lo económico, lo social y lo ambiental, reconociendo los intereses de los distintos grupos con los que se relaciona y buscando la preservación del medio ambiente y la sustentabilidad de las generaciones futuras". Su visión de negocios debe comprender el respeto por las personas, los valores éticos, la comunidad y el medioambiente con la gestión misma de la empresa, independientemente de los productos o servicios que ésta ofrece, del sector al que pertenece, de su tamaño o nacionalidad.

La presente investigación se desarrolló en una institución educativa privada a nivel superior, de la zona Norte en Monterrey, N.L., dentro de la renovación de los programas educativos 2017-2020 se agrega el apartado de elaboración de proyectos integradores de la unidad académica, en el cuál el alumno como parte de su calificación y para acreditar el curso, deberá participar en un proyecto de integración académica socialmente responsable. Se I гпAIV-LA 
ESTUDIO DE LOS COMPORTAMIENTOS DE CIUDADANÍA ORGANIZACIONAL EN LOS PROYECTOS UNIVERSITARIOS DE RESPONSABILIDAD SOCIAL

trabajó con un grupo de alumnos de segundo cuatrimestre de la materia de administración de empresas, que como fin medible y cuantificable de su proyecto fue cumplir en tiempo y forma con el objetivo social encomendado al equipo; de esta manera, se logró involucrar y sensibilizar al estudiante con su comunidad.

En esta investigación se relacionan los conceptos teóricos de ciudadanía organizacional con el rendimiento individual y de equipo para lograr la generación de responsabilidad social de manera natural e innata, observando como los comportamientos o dimensiones de ciudadanía organizacional que son conductas reconocidas formalmente en los sistemas de trabajo pero que están inmersas en las conductas de la persona, influyen en el rendimiento del equipo, otro oponente que describe a la ciudadanía organizacional es el aspecto discrecional, este observa actividades que de forma voluntaria el individuo desarrolla, generando comportamientos o conductas específicas en su desempeño y que no están requeridas en el rol o papel establecido en su equipo de trabajo, pero que son necesarias en su relación de equipo de trabajo (Organ, 1988).

El rendimiento individual o de equipo se observa en el cumplimiento en tiempo y forma de la meta planteada al inicio del período escolar, así como el equipo solucionó los problemas que se le presentaron y redefinieron sus estrategias para el logro de la misma.

\section{MARCO TEÓRICO}

\section{Comportamiento de ciudadanía organizacional}

Los comportamientos de ciudadanía organizacional parten del campo de la Psicología Industrial u Organizacional, en la cual se analiza el impacto de la conducta de los individuos, los grupos y las estructuras en las que se distinguen las organizaciones. Este comportamiento es definido como conductas que son manifestadas de manera individual, las cuáles son discrecionales y no son reconocidas por el sistema formal de la organización, pero que son necesarias, ya que en su conjunto promueven el funcionamiento eficiente y efectivo de la organización (Organ, 1988).

Organ (2006) citado por Terán, et. al., (2016) menciona que: la ciudadanía organizacional se caracteriza por ejecutar acciones que no se encuentran manifestadas en el perfil del puesto de un empleado; no obstante, este empleado realiza dichas actividades para beneficio de su organización, cubriendo un espacio en blanco de un ambiente laboral que es imprescindible para el buen desenvolvimiento de las actividades organizacionales. Estas manifestaciones están dirigidas hacia los compañeros o hacia la propia organización en sí, no están I IKAIN-CALAKES IMI. IVI., PAKIIUA-PUENIL A., KUUKIGULL-GAKLA B. N. 
ESTUDIO DE LOS COMPORTAMIENTOS DE CIUDADANÍA ORGANIZACIONAL EN LOS PROYECTOS UNIVERSITARIOS DE RESPONSABILIDAD SOCIAL

contempladas en su rol o papel y tampoco están establecidas en el puesto, esta conducta va más allá del puesto para el que fue contratado el individuo, por tal circunstancia no está especificado en sus obligaciones contractuales de trabajo.

Para efecto de esta investigación, se consideraron las dimensiones estudiadas en las investigaciones realizadas por Organ, Podsakoff y MacKenzie, en el 2006, en donde identificaron siete dimensiones de comportamiento ciudadano (Terán, 2012) las cuáles se describen a continuación:

1.- Ayuda. Dándose de forma voluntaria, provoca armonía en las relaciones interpersonales entre los miembros de la organización, al apoyar a los compañeros en situaciones del trabajo o no necesariamente del mismo trabajo, ya que es el asistir, ayudar o apoyar.

2.- Lealtad organizacional. Es el defender la imagen y reputación de la empresa dentro y fuera de ella; es el apoyar y colaborar con la organización; promocionarla y proteger los intereses de la empresa, así como confiar en ella en situaciones complicadas.

3.- Cumplimiento organizacional. Esta dimensión describe cumplir con elementos imperantes de la organización como la atención, la puntualidad, así como cuidar los recursos de la empresa, al aceptar y cumplir en todo momento las regulaciones y procedimientos organizacionales.

4.- Iniciativa individual. Esta dimensión describe la proactividad en las decisiones, el dinamismo, la creatividad y la innovación que se genera y que favorecen la imagen de la organización.

5.- Deportivismo. Es la voluntad de tolerar los inconvenientes presentados en la actividad organizacional, con una actitud de positivismo.

6.- Virtud cívica. Es el reconocer o sentir el colaborador que es parte de la organización y aceptan las obligaciones y responsabilidades que esto conlleva.

7.- Auto desarrollo. Es el compromiso voluntario de los trabajadores por superarse, capacitarse y mantenerse actualizado sin considerar que es una obligación, sino que crece a la vez con su organización. 
ESTUDIO DE LOS COMPORTAMIENTOS DE CIUDADANÍA ORGANIZACIONAL EN LOS PROYECTOS UNIVERSITARIOS DE RESPONSABILIDAD SOCIAL

\section{Responsabilidad social}

Las grandes organizaciones en el orbe han incorporado a sus estrategias de negocio la RSE como un elemento diferenciador y como ventaja competitiva, con resultados económicos positivos. Asimismo, son muchos los estudios, a nivel internacional, que comprueban la correlación positiva entre el crecimiento económico y el comportamiento socialmente responsable de la empresa (García, 2014). Los ciudadanos esperan hoy, que la empresa sea parte de un cambio positivo en sus comunidades, contribuyendo de manera sustentable al desarrollo de la sociedad.

Asumir la RSE en las organizaciones es una tarea que implica decisiones de liderazgo, a fin de superar problemas como la baja cultura cívica, la corrupción, la falta de marcos legales, autoritario rol de mando y otros que han impedido a la empresa desarrollarse como ciudadano responsable con su entorno, por lo que Cajiga (2004) propone compromisos básicos de acción:

- Fomentar el desarrollo y bienestar social de las comunidades en las que opera.

- Considerar las necesidades del entorno social del negocio en la toma de decisiones y colaborar en su solución.

- Hacer públicos sus compromisos con la sociedad y medir los logros alcanzados.

- Vivir conforme a esquemas de liderazgo participativo, solidario, de servicio y respetuoso de la dignidad humana, actuando con base en un código de ética.

- Contribuir al desarrollo humano y profesional de la comunidad laboral de la empresa y de sus familias.

- Apoyar alguna causa social afín a la actividad que desarrolle la empresa.

- Respetar, preservar y regenerar el medio ecológico en todos y cada uno de los procesos de operación, comercialización y actividades que realice.

De lo anterior, se concluye que la RSE comprende cuatro ámbitos básicos y estratégicos; los cuales a su vez se evidencian en toda actividad de la empresa (Arévalo, 2015):

- Ética y gobernabilidad empresarial.

- Calidad de vida en la empresa (dimensión social del trabajo).

- Vinculación y compromiso con la comunidad y su desarrollo.

- Cuidado y preservación del medioambiente. 
ESTUDIO DE LOS COMPORTAMIENTOS DE CIUDADANÍA ORGANIZACIONAL EN LOS PROYECTOS UNIVERSITARIOS DE RESPONSABILIDAD SOCIAL

\section{Rendimiento individual y de equipo}

El rendimiento académico se refiere a la evaluación del conocimiento adquirido en el ámbito escolar. Un estudiante con buen rendimiento académico, es aquél que obtiene buenas calificaciones en los exámenes que debe rendir a lo largo de un curso. La complejidad del rendimiento académico inicia desde su conceptualización, algunas veces se le denomina como aptitud escolar, desempeño académico o rendimiento escolar, pero generalmente las diferencias sólo tienen que ver con cuestiones semánticas, ya que generalmente, en los textos la vida escolar y la experiencia docente, son utilizadas como sinónimos (Navarro, 2003).

El rendimiento académico supone la capacidad del alumno para responder a los estímulos educativos; es una medida de las capacidades que expresa lo que éste ha aprendido a lo largo del proceso formativo. El rendimiento escolar es un nivel de conocimientos demostrado en un área o materia, comparado con la norma de edad y nivel académico. Por tanto, el rendimiento del alumno debería ser entendido a partir de sus procesos de evaluación; sin embargo, la simple medición y/o evaluación de los rendimientos alcanzados por los alumnos no provee por sí misma todas las pautas necesarias para la acción destinada al mejoramiento de la calidad educativa (Jiménez, 1994).

Existen distintos factores que inciden en el rendimiento académico, desde la dificultad propia de algunas asignaturas, hasta la gran cantidad de exámenes que pueden coincidir en una fecha, pasando por la amplia extensión de ciertos programas educativos, son muchos los motivos que pueden llevar a un alumno a mostrar un pobre rendimiento académico. Otras cuestiones están directamente relacionadas al factor psicológico, como la poca motivación, el desinterés o las distracciones en clase, que dificultan la comprensión de los conocimientos impartidos por el docente y termina afectando al rendimiento académico (Huaylupo, 2004).

Si se pretende conceptualizar el rendimiento académico a partir de la evaluación, es necesario considerar no solamente el desempeño individual del estudiante sino la manera como es influido por el grupo de pares, el aula o el propio contexto educativo. Navarro (2003), (citando a Cominetti y Ruiz, 1997) enfatiza sobre algunos factores del rendimiento: las expectativas de familia, docentes y los mismos alumnos con relación a los logros en el aprendizaje, reviste especial interés debido a que pone al descubierto el efecto de un conjunto de prejuicios, actitudes y conductas que pueden resultar beneficiosos 0 desventajosos en la tarea escolar y sus resultados. Asimismo, que el rendimiento de los TERÁN-CÁZARES M. M., PARTIDA-PUENTE A., RODRÍGUEZ-GARZA B. N. 
ESTUDIO DE LOS COMPORTAMIENTOS DE CIUDADANÍA ORGANIZACIONAL EN LOS PROYECTOS UNIVERSITARIOS DE RESPONSABILIDAD SOCIAL

alumnos es mejor, cuando los maestros manifiestan que el nivel de desempeño y de comportamientos escolares del grupo es adecuado.

Ramírez (2011) cita a Paulo Freire y señala que "nadie forma a nadie, nadie se forma solo, los hombres se forman entre sí mismos mediatizados por el mundo". Para esto, es menester que las personas sean formadas para vivir en sociedad, para poder compartir el compromiso de transformar su conciencia y sobre todo las circunstancias que las crearon, logrando diferenciar lo positivo de lo negativo que se encuentra en el medio que lo rodea. La tesis de Freire (1970), hace referencia a que el éxito de la formación se basa en el diálogo, para que esto ocurra, es necesario el amor, la humildad, la esperanza y el pensamiento crítico.

\section{METODOLOGÍA}

La investigación es exploratoria, descriptiva, correlacional y explicativa, con un corte transversal, considerando el estudio de manera cuantitativa, cualitativamente y no experimental. El universo de la presente investigación la integran los estudiantes universitarios que cursan el primer cuatrimestre en la escuela de negocios de una Universidad Privada en Monterrey, N.L., los cuales participaron en proyectos integradores de responsabilidad social, conformando una muestra de 68 estudiantes de un total de 82 estudiantes inscritos en el área, dicha muestra fue elegida de acuerdo a la totalidad de los estudiantes que culminaron los proyectos de responsabilidad social.

De acuerdo a la revisión conceptual, se plantea la siguiente hipótesis:

H1.- Los factores que inciden y tienen un efecto positivo en el rendimiento individual y de equipo de los comportamientos de ciudadanía organizacional de los alumnos universitarios de las escuelas de negocios en proyectos integradores de responsabilidad social son:

A: Ayuda, D: Deportivismo, L: Lealtad, C: Cumplimiento, I: Iniciativa, V: Virtud cívica y AE: Autodesarrollo.

El análisis conceptual, permite inferir que los comportamientos de ciudadanía organizacional que demostraron los estudiantes a través de las dimensiones de ayuda, iniciativa, deportivismo, lealtad, cumplimiento, virtud cívica y autodesarrollo tienen un efecto positivo en el rendimiento individual y del equipo en las actividades que desarrollaron para el cumplimiento del objetivo o meta social de su proyecto de integración. 
ESTUDIO DE LOS COMPORTAMIENTOS DE CIUDADANÍA ORGANIZACIONAL EN LOS PROYECTOS UNIVERSITARIOS DE RESPONSABILIDAD SOCIAL

Es importante mencionar, que se cumplió con la objetividad a través de la estandarización de la aplicación de la encuesta, ya que se consideró equitativo el porcentaje de participación de los grupos participantes, se aplicó en los horarios y lugares académicos, se les brindó la misma atención personal para resolver sus dudas, se entregó la encuesta de forma física impresa en un solo color de tinta.

En ese sentido, la aplicación se desarrolló en 4 etapas:

En la primera etapa, se desarrolló una revisión bibliográfica, de las variables analizadas, observando los constructos que midieran las variables propuestas, se tradujeron y adaptaron, con el apoyo de traductores especialistas avalados en el campo de las ciencias sociales.

En una segunda etapa, se realizó una validez de apariencia, a través de un grupo de expertos, se revisó y reestructuró la redacción de cada uno de los ítems, de esta manera se determinó cubrir la necesidad de una validez aparente y garantizar que cada ítem tuviera un aspecto general de aceptabilidad, de acuerdo a la percepción de la población de estudio.

En la tercera etapa, se desarrolló una prueba piloto, aplicando 20 encuestas en una muestra muy similar a la población de estudio, con la finalidad de aplicar el análisis de confiabilidad del instrumento a través del Alfa de Cronbach, la cual superó el 0.7 en su análisis, el instrumento que se aplicó fue con un total de 84 preguntas, en la primer sección se midió los comportamientos de ciudadanía organizacional, en la segunda sección se midió el rendimiento individual y de equipo y en la tercera sección se pidió información general del perfil del encuestado. En el instrumento se utilizó una escala de Likert, en donde 1 es nada de acuerdo, 2 poco de acuerdo, 3 ligeramente de acuerdo, 4 de acuerdo y 5 totalmente de acuerdo.

La variable dependiente es el rendimiento individual y de equipo y la variable independiente son los comportamientos de Ciudadanía Organizacional, integrados por las dimensiones de ayuda, iniciativa, lealtad, cumplimiento, virtud cívica y autodesarrollo.

La cuarta etapa, consistió en redefinir el instrumento al eliminar los ítems que en la 3era etapa obtuvieron resultados que disminuían nuestra Alfa de Cronbach; cabe mencionar, que el instrumento original tenía 84 ítems, posterior al proceso anterior quedaron 64 ítems, se rediseñó el instrumento y se encuestó a la población objetivo, la cual consistía en los estudiantes que habían participado en proyectos integradores de responsabilidad social. 
ESTUDIO DE LOS COMPORTAMIENTOS DE CIUDADANÍA ORGANIZACIONAL EN LOS PROYECTOS UNIVERSITARIOS DE RESPONSABILIDAD SOCIAL

Al analizar los resultados obtenidos, aplicadas las 68 encuestas, se observó que los grados de correlación que se tienen entre los ítems de cada uno de los constructos, la validez y confiabilidad del instrumento de medición considerando las variables independientes y dependientes muestran un análisis de las Alfas de Cronbach satisfactorias, todas por encima de 0.6 (Cronbach, 1951) (ver tabla 1).

Tabla 1

Alfa de Cronbach para cada una de las variables

\begin{tabular}{lcc}
\hline Variables & Ítems originales & Alfa de Cronbach \\
\hline DEPENDIENTES & 10 & 0.816 \\
$\quad$ R $=$ Rendimiento Individual y de Equipo & & \\
INDEPENDIENTES & 4 & 0.856 \\
A $=$ Ayuda & 4 & 0.823 \\
D = Deportivismo & 3 & 0.772 \\
L = Lealtad & 5 & 0.689 \\
C = Cumplimiento & 3 & 0.871 \\
I = Iniciativa & 3 & 0.610 \\
V = Virtud cívica & 3 & 0.767 \\
AE = Autodesarrollo & &
\end{tabular}

Fuente: Elaboración propia.

De acuerdo a estos resultados, se observa que las preguntas de cada uno de constructos no están correlacionadas, son totalmente independientes una de la otra, y que una no depende de la otra. Por lo tanto, tenemos validez de la información que se recabo con el instrumento.

\section{RESULTADOS}

\section{Modelo}

Los resultados que se obtuvieron en la regresión lineal múltiple de las variables independientes, A: Ayuda, D: Deportivismo, L: Lealtad, C: Cumplimiento, I: Iniciativa, V: Virtud Cívica y $A E$ : Autodesarrollo, en relación a las variables dependientes, $R=$ Rendimiento Individual y de Equipo; son los que se describen a continuación.

Los valores que se observan en la Durbin-Watson, comprueba la independencia de residuales y muestra la presencia de auto correlación de primer orden en los residuales en una regresión, en el presente estudio el valor Durbin-Watson es de 2.062 (ver tabla 2), lo cual define que no existe auto correlación en el modelo de estudio, considerando una 
ESTUDIO DE LOS COMPORTAMIENTOS DE CIUDADANÍA ORGANIZACIONAL EN LOS PROYECTOS UNIVERSITARIOS DE RESPONSABILIDAD SOCIAL

regresión lineal múltiple dentro del valor aceptado (VIF) de un rango establecido entre 1.164 a 1.659 (véase tabla 4), con el coeficiente de correlación aceptado ( $r$ ) de 0.715 para el modelo de la presente investigación, el coeficiente de determinación de $\left(\mathrm{r}^{2}\right)$ corresponde a 0.511 (ver tabla 2).

Tabla 2

Resultados de la regresión lineal múltiple

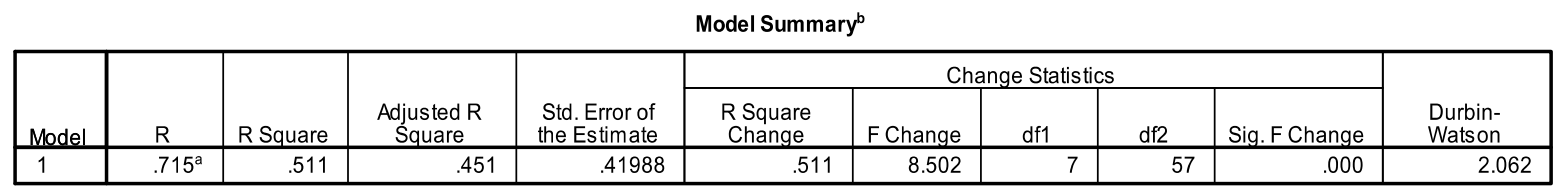

a. Predictors: (Constant), AE, A, L, D, V, C, I

b. Dependent Variable: $R$

Fuente: SPSS-V18.

\section{Análisis de Varianza}

El análisis de varianza ANOVA, compara las medias de cada variable para definir que existe una independencia entre las variables independientes, por lo que se deduce que el modelo es aceptado, en la tabla 3, muestra la independencia contra las otras variables por tener un valor inferior del $5 \%$ de la significancia.

Tabla 3

Análisis de Varianza ANOVA

ANOVA $^{b}$

\begin{tabular}{|ll|r|r|r|r|c|}
\hline Model & & $\begin{array}{c}\text { Sum of } \\
\text { Squares }\end{array}$ & df & Mean Square & \multicolumn{1}{c|}{$\mathrm{F}$} & Sig. \\
\hline 1 & Regression & 10.493 & 7 & 1.499 & 8.502 & $.000^{\mathrm{a}}$ \\
& Residual & 10.049 & 57 & .176 & & \\
& Total & 20.542 & 64 & & & \\
\hline
\end{tabular}

a. Predictors: (Constant), AE, A, L, D, V, C, I

b. Dependent Variable: $R$

Fuente: SPSS-V18.

\section{T-Student}

Con base a los resultados obtenidos a las significancias de la T-student referido en la tabla 4 , se observa un error estimado menor al $5 \%$ donde se pueden definir cada una de las variables independientes que impactan significativamente en el rendimiento individual y 
ESTUDIO DE LOS COMPORTAMIENTOS DE CIUDADANÍA ORGANIZACIONAL EN LOS PROYECTOS UNIVERSITARIOS DE RESPONSABILIDAD SOCIAL

grupal del comportamiento de ciudadanía organizacional de los alumnos universitarios de las escuelas de negocios en proyectos integradores de responsabilidad social.

Tabla 4

Significancia de la T-Student

\begin{tabular}{|c|c|c|c|c|c|c|c|c|c|c|c|}
\hline \multicolumn{12}{|c|}{ Coefficients $^{\mathrm{a}}$} \\
\hline \multirow[b]{2}{*}{ Mode } & & \multicolumn{2}{|c|}{ Unstandardized Coefficients } & \multirow{2}{*}{$\begin{array}{c}\text { Standardized } \\
\text { Coefficients }\end{array}$} & \multirow[b]{2}{*}{$t$} & \multirow[b]{2}{*}{ Sig. } & \multicolumn{3}{|c|}{ Correlations } & \multicolumn{2}{|c|}{ Collinearity Statistics } \\
\hline & & B & Std. Error & & & & Zero-order & Partial & Part & Tolerance & VIF \\
\hline \multirow[t]{8}{*}{1} & (Constant) & .856 & .542 & & 1.579 & .120 & & & & & \\
\hline & A & .129 & .065 & .205 & 1.995 & .051 & .278 & .255 & .185 & .812 & 1.231 \\
\hline & $\mathrm{D}$ & -.013 & .057 & -.023 & -.224 & .823 & -.245 & -.030 & -.021 & .808 & 1.237 \\
\hline & L & .155 & .074 & .211 & 2.107 & .040 & .362 & .269 & .195 & .859 & 1.164 \\
\hline & C & .072 & .079 & .099 & .916 & .364 & .298 & .120 & .085 & .732 & 1.366 \\
\hline & I & .251 & .083 & .363 & 3.040 & .004 & .597 & .374 & .282 & .603 & 1.659 \\
\hline & V & .039 & .089 & .049 & .442 & .660 & .389 & .058 & .041 & .685 & 1.459 \\
\hline & $\mathrm{AE}$ & .146 & .069 & .230 & 2.105 & .040 & .462 & .269 & .195 & .717 & 1.395 \\
\hline
\end{tabular}

a. Dependent Variable: $R$

Fuente: SPSS-V18.

En el presente estudio, la ecuación de regresión lineal para la variable dependiente $\mathrm{R}=$ Rendimiento Individual y de Equipo, se muestra a continuación:

Rendimiento Individual y de Equipo $=0.856+0.129$ Ayuda

+0.155 Lealtad

+0.251 Iniciativa

+0.146 Autodesarrollo $+\varepsilon$

El modelo de la presente investigación, se explica en un $71.5 \%$. El impacto en el rendimiento individual y de grupo de los estudiantes universitarios de las escuelas de negocios en proyectos integradores de responsabilidad social, la Ayuda como factor del comportamiento de ciudadanía organizacional, impacta en un $12.9 \%$, mientras que la Lealtad impacta en un $15.5 \%$, la Iniciativa impacta en un $25.1 \%$ y el Autodesarrollo impacta en un $14.5 \%$.

\section{CONCLUSIONES}

Para lograr el rendimiento individual y de grupo de los estudiantes universitarios de las escuelas de negocios en proyectos integradores de responsabilidad social, la Ayuda como factor del comportamiento de ciudadanía organizacional, deberá lograrse a través del apoyo 
ESTUDIO DE LOS COMPORTAMIENTOS DE CIUDADANÍA ORGANIZACIONAL EN LOS PROYECTOS UNIVERSITARIOS DE RESPONSABILIDAD SOCIAL

de unos a otros en el trabajo para beneficio del grupo, involucrarse con el equipo de trabajo y ayudarse en el aprendizaje y en las responsabilidades de trabajo.

Para lograr la Lealtad como factor del comportamiento de ciudadanía organizacional e impacte en el rendimiento individual y de equipo, la organización deberá protegerse contra amenazas exteriores, recomendar la organización para trabajar dentro de ella y defenderla cuando es criticada por sus propios empleados.

Para lograr la Iniciativa como factor del comportamiento de ciudadanía organizacional e impacte en el rendimiento individual y de equipo, se deberá motivar a los demás para que externen sus ideas y opiniones, animar a los demás para que hagan su trabajo de una manera diferente y más efectiva.

Para lograr el Autodesarrollo como factor del comportamiento de ciudadanía organizacional e impacte en el rendimiento individual y de equipo, el estudiante debe pensar que el crecimiento personal es más importante que la posición de trabajo, debe sentir que el incremento en el trabajo no es problema cuando el pago por este refleja su crecimiento personal, además de percibir que sus salarios y prestaciones compensan adecuadamente sus esfuerzos.

\section{REFERENCIAS BIBLIOGRÁFICAS}

Arévalo, J. G., Bayona, R. A. y Rico, D. W. (2015). Responsabilidad social empresarial e innovación: Una mirada desde las tecnologías de la información y comunicación en organizaciones. Clío América, 9(18), 180-189. Recuperado de http://dx.doi.org/10.21676/23897848.1535

Cajiga, J. F. (2004). El concepto de Responsabilidad Social Empresarial. pp.35. México: CEMEFI. Recuperado de https://www.cemefi.org/esr/images/stories/pdf/esr/concepto_esr.pdf

García, M. y Peláez, J. D. (2014). Responsabilidad social empresarial y gestión humana: una relación estratégica aplicada desde un modelo explicativo. Entramado, 10(2) 90-111. Recuperado de http://www.redalyc.org/articulo.oa?id=265433711007

Huaylupo, J. (2004). La evaluación del desempeño ¿Un enfoque individual del trabajo colectivo, o una visión individualista del trabajo social? Fiscalización y Gestión Pública, 2(2), 73-84. Universidad de Costa Rica. 
ESTUDIO DE LOS COMPORTAMIENTOS DE CIUDADANÍA ORGANIZACIONAL EN LOS PROYECTOS UNIVERSITARIOS DE RESPONSABILIDAD SOCIAL

Jiménez, M. (1994). Competencia social: intervención preventiva en la escuela. Infancia y Sociedad. Revista de Estudios, (24), 21-48. ISSN 1131-5954.

Navarro, R. (2003). El rendimiento académico: concepto, investigación y desarrollo. Revista Electrónica Iberoamericana sobre Calidad, Eficacia y Cambio en Educación, 1(2), 1-15. Recuperado de http://www.ice.deusto.es/RINACE/reice/vol1n2/Edel.pdf

Organ, D. (1988). Organizational citizenship behavior: the good soldier syndrome, lexington books. Massachusetts. 133.

Organ, D., Podsakoff, P. y Mackenzie, S. (2006). Organizational citizenship behavior: its nature, antecedents, and consequences. Sage Publications, Inc. Thousand Oaks, California.

Rodríguez, A., Cruz, C. y López, E. (2013). Educación para la Sustentabilidad: Una experiencia chontal. Horizonte Sanitario, 12(3), 119-121. ISSN: 1665-3262. Recuperado de http://www.redalyc.org/pdf/4578/457845145008.pdf

Ramírez, M., Devia, R. E. y León, R. A. (2011). Pobreza y rendimiento escolar: estudio de caso de jóvenes de alto rendimiento. Educere, 15(52), 663-672. Recuperado de http://www.redalyc.org/articulo.oa?id=35622379013

Terán, M. y Mendoza, J. (2012). La influencia de los rasgos de la cultura de la organización en los comportamientos de ciudadanía organizacional. XVII Congreso Internacional de Contaduría, Administración e Informática, UNAM. Recuperado de: http://congreso.investiga.fca.unam.mx/docs/xvii/docs/E09.pdf

Terán, M. M., Partida, A., Blanco, M. y Rodríguez, B. N. (2016). La cultura organizacional como influencia en la generación de comportamientos de ciudadanía organizacional: estudio de los laboratoristas de una universidad pública en la zona norte. Vinculatégica, 2(1), ISSN: 2448-5101.

UNESCO (2012). Organización de las Naciones Unidas para la Educación, la Ciencia y la Cultura. Educación para el Desarrollo Sostenible. pp. 53. ISBN 978-92-3-0010775. París: UNESCO. Recuperado de: http://unesdoc.unesco.org/images/0021/002167/216756s.pdf

UNESCO (2016). Organización de las Naciones Unidas para la Educación, la Ciencia y la Cultura. Recuperado de: http://www.unesco.org/new/es/education/themes/leadingthe-international-agenda/education-for-sustainable-development/sustainabledevelopment/ 